\title{
Molecular Identification of Sarcocystis spp. in Sheep and Cattle by PCR-RFLP from Southwest of Iran
}

\author{
Mahmoud Rahdar, ${ }^{1,2}$, and Tahereh Kardooni ${ }^{2}$ \\ ${ }^{1}$ Infectious and Tropical Diseases Research Center, Health Research Institute Ahvaz Jundishapur University of Medical Sciences, Ahvaz, Iran \\ ${ }^{2}$ Department of Parasitology, Faculty of Medicine, Ahvaz Jundishapur University of Medical Sciences, Ahvaz, IR Iran \\ "Corresponding author: Mahmoud Rahdar, Department of Parasitology, Faculty of Medicine, Ahvaz Jundishapur University of Medical Sciences, Ahvaz, IR Iran. Tel: \\ +98-9166153521, Fax: +98-6133332036, E-mail: rahdar-m@ajums.ac.ir
}

Received 2017 May 09; Accepted 2017 June 24.

\begin{abstract}
Background: Sarcocystis species are obligatory intracellular parasites of many vertebrate hosts. Some pathogen species cause major economic loss and hygienic problems in the animal and human population, respectively.

Objectives: The goal of the current study was conducted to identify Sarcocystis species in meat-producer animals and to evaluate the risk of transmission of parasites after consumption of infected meat by humans.

Methods: Fifty samples of sheep and cattle muscles were collected from the abattoir. The samples were collected from the heart, tongue, diaphragm, and skeletal muscles. The PCR method was used for amplifying the $18 \mathrm{~S}$ ribosomal RNA gene for distinguish Sarcocystis species using 2 primers and 3 restricted enzymes including Hinf, Mbo1, and EcoR1.

Results: The results showed that all cattle samples were infected by Sarcocystiscruzi (100\%) and sheep samples were contaminated by S. tenella (80\%) as well as S. capracanis (20\%). No human Sarcocystis species were detected.

Conclusions: Meat-producer animals are infected by S. cruzi as well as S. tenella and the consumption of infected meat is not important for human sarcocystosis in this area.
\end{abstract}

Keywords: Sheep, Cattle, Polymerase Chain Reaction, RFLP, Sarcocystis

\section{Background}

Many protozoan parasites can transmit to humans via ingestion of contaminated meat with various types of parasite cysts. In point of view, Toxoplasma, Sarcocystis, and Trichenella are some examples of these agents. Sarcocystis species are obligatory apicomplexan intracellular parasites in a wide range of vertebrate including mammals, birds, and reptiles. The parasite has 2 hosts, which spend their sexual life cycle in definitive host (predator) and asexual life cycle in intermediate host (prey). Sexual life takes place in an intestinal epithelial cell of final host, which finally produce oocysts and passes from feces and contaminate environment. In the intermediate host, parasite proliferates in various types of cells by merogony division and finally produces macroscopic or microscopic cyst in muscles as well as brain, according to species $(1,2)$. Approximately, 150 species of Sarcocystis with variety of clinical signs were introduced in the world (3). There are 4 predominant Sarcocystis species in sheep with worldwide distribution including, Sarcocystis tenella, S. gigantea (S. ovifelis), S. arieticanis, and S. medusiformis (4).

There are 3 species of Sarcocystis in cattle including S. bovifelis (S. hirsuta), S. cruzi (S. bovicanis), and S. hominis (S. bovihominis) (5). The severity of clinical signs in domesticated animals depends on the species of parasite and number of digested oocysts. Clinical signs included fever, anorexia, tachypnoe, tachycardia, anemia, encephalitis, encephalomyelitis, and hemorrhage, which can cause death in infected animals (6). The other feature of infection is abortion and fetal death in pregnant sheep (7). Sarcocystis tenella is a pathogen species in sheep; However, S. ovifelis is not. Additional economic losses are a condemnation of whole or partly carcasses in a slaughterhouse, reduction of animal products such as milk, meat, wool, and decreasing fertility (8). Economic losses in Spain have been estimated 20 million Euros annually due to condemnation of carcasses (9).

Humans can be infected as intermediate and final hosts by S. hominis, S. porcihuminis, and S. lindemanni (5). Biopsy or necropsy finding revealed that human serves as an intermediate host for at least 7 species of Sarcocystis (3). Many infections in humans are asymptomatic, however, some clinical signs in infected individuals were reported, which include vomiting, nausea, acute or chronic enteritis according to the species, and a number of ingested cyst (3). The other aspect of human sarcocystosis is muscular sarcocystosis. The most muscular sarcocystosis patients are asymptomatic and only 10 cases were reported with acute inflammation of muscles in the world $(10,11)$. The disease 
is endemic in some part of tropical country, especially in Malaysia (12).

Elevation of hepatic enzymes ALT (alanine aminotransferase), AST (aspartate aminotransferase), CRP (C reactive protein), ESR (erythrocyte sedimentation rate), LDH (lactic dehydrogenase), CK (creatinine phosphokinase), and Eosinophilia has been reported in eosinophilic myositis caused by sarcocystosis $(13,14)$. The conventional diagnosis of different species of Sarcocystis is based on the structure study of the cyst wall by using light or transmission electron microscopic (15). This method is not very precise because of existence changes of cysts during tissue processing and age of cyst (3).

\section{Objectives}

The goal of this study was conducted to identify the species of Sarcocystis by RFLP-PCR and nucleotide sequencing method in sheep and cattle to identify public health problem in human community.

\section{Methods}

\subsection{Sampling}

Fifty samples were collected from the diaphragm, heart, skeletal muscles of sheep and cattle carcasses (each animal 25 samples) in industrial abattoir of Ahvaz, and 50 mg of each sample were considered for DNA extraction using commercial DNA extract kit (QIAamp DNA Mini Kit, Qiagen, Germany). DNA was extracted according to manufacture instructions and stored in -20 centigrade until used.

\subsection{PCR-RFLP}

In this study 2 pair primers were used. A variable region of the $18 \mathrm{~S}$ ribosomal RNA gene was used as a good marker for determining sheep Sarcocystis species $(16,17)$, which included $18 \mathrm{~S}$ forward (5/ GGA TAA CCG TGG TAA TTC TAT G3/) and $18 \mathrm{~S}$ reverse (5/ TCC TAT GTC TGG ACC TGG TGA $\mathrm{G} 3 /$ ). The primer amplify a part of 18 s ribosomal gene with 1100 bp length (18). The PCR protocol was performed 5 minutes primary denaturation at $94^{\circ} \mathrm{C}$ follow by 35 cycles of $94^{\circ} \mathrm{C}$ for 1 minute, $50^{\circ} \mathrm{C}$ for 1 minute, and $72^{\circ} \mathrm{C}$ for 90 seconds, as well as a final extension of $72^{\circ} \mathrm{C}$ for 10 minutes (19). The final volume of PCR reaction was $25 \mu \mathrm{L}$ including $5 \mu \mathrm{L}$ of the sample DNA, 20 pmol of each primer,12.5 $\mu \mathrm{L}$ of PCR Master Mix (Ampliqon, Denmark), and $5.5 \mu \mathrm{L}$ distillated water.

The second pair primer was designed for detecting cattle Sarcocystis species, which almost amplify a 600 bp segment. The primer was Sar F 5/ GCA CTT GAT GAA TTC TGG CA 3/ and Sar R 5/ CAC CAC CCA TAG AAT CAA G 3/ (20). The
PCR steps included $94^{\circ} \mathrm{C}$ for 5 minutes followed by 40 cycles of $94^{\circ} \mathrm{C}$ for 2 minutes, annealing at $55^{\circ} \mathrm{C}$ for 1 minute, and extension step $72^{\circ} \mathrm{C}$ for 90 seconds, followed by a final extension step at $72^{\circ} \mathrm{C}$ for 5 minutes. The final volume of PCR reaction was $25 \mu \mathrm{L}$ including $3 \mu \mathrm{L}$ of the sample DNA, $20 \mathrm{pmol}$ of each primer, and $12.5 \mu \mathrm{L}$ of PCR Master Mix and $7.5 \mu \mathrm{L}$ distillated water. The PCR bands appear by using gel electrophoresis in 1\% agarose, ethidium bromide, and gel documentation apparatus.

The PCR products were digested using the restriction fragment length polymorphism (RFLP) method by 3 nucleotide enzymes including Haemophilus influenza Rf endonuclease enzyme (Hinf), Moraxella bovis endonuclease enzyme (Mbo1), and Escherichia coli RY13 endonuclease enzyme (EcoR1). The RFLP reaction was carried out by $5 \mu \mathrm{L}$ PCR product, 10 unit of each enzyme, and buffer. The reaction are incubated 24 hours at $37^{\circ} \mathrm{C}$. Eight samples of PCR yields of sheep and cattle (each one 4 samples) was sent to Bioneer company South Korea for nucleotide sequencing. The nucleotide sequences were compared with other registered nucleotide sequencing in the National Center for Biotechnology Information (NCBI).

\section{Results}

Fifty samples of sheep and cattle (each 25 samples) were selected for PCR-RFLP molecular technique. These samples were previously examined for Sarcocystis species contamination by using the digestive method with HCL and pepsin; the result showed that all samples were infected by Sarcocystis (21). Two pair primers were selected to identify Sarcocystis species in sheep and cattle. PCR finding presented that a nucleotide segment with 600 base per was amplified for cattle samples (Figure 1). The comparison of nucleotide sequencing with other registered nucleotides sequencing in NCBI confirmed that they belong to S. cruzi with $99 \%$ homology (Figure 2 ). There is no difference between isolates when aligned together with Mega 6 software (Figure 2). The use of 3 restricted enzymes for the RFLP study presented that Eco1enzyms cannot break down the PCR yield. The Mbo restricted enzyme can divide the PCR product to 2 bands approximately 300 and 330 base pair. The Hinf enzymes produced 2 bands including 550 and $50 \mathrm{bp}$ (Figure 3 ).

The PCR of $18 \mathrm{~S}$ ribosomal RNA gene in sheep samples amplified a 1100 base pair and 900 bp nucleotide (Figure 4 ), which pertained to S. tenella and S. capriovis, respectively. The comparison of nucleotide sequence with other nucleotide in NCBI revealed that they have 100\% homology with S. tenella and S. capricanis. The use of 3 restricted enzymes for the RFLP study on S. tenella PCR presented that 
Figure 1. The Electrophoresis of the PCR Product of Ribosomal Gene of Sarcocystis in Cattle Samples That Presented a Band with 600 bp Belonged to S. cruzi

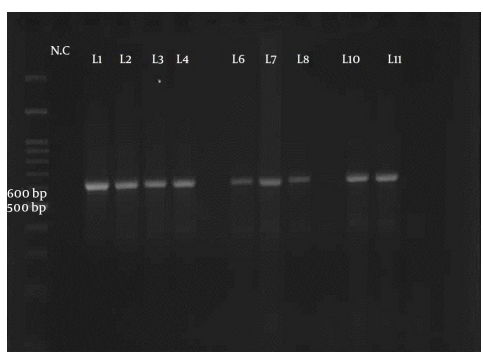

(N.C negative control, line 1-4, 6-8 and 10 - 11 positive).

Eco1enzyms break down the PCR yield to 2 fractions, including 600 and 400 BP (Figure 5). The Mbo restricted enzyme can divide the PCR yield to 3 bands approximately 750, 250, and 150 base pair. The Hinf enzymes produced 2 bands including 750 and 400 (Figure 5). The comparison of nucleotide sequences of PCR product of Sarcocystis in sheep samples with NCBI data revealed that $100 \%$ homology for S. tenella and 99\% for S. capricanis (Figure 6).

\section{Discussion}

Some Sarcocystis species can cause clinical signs in the human population. Men serve as intermediated hosts with myositis symptoms and a definitive host with gastrointestinal symptoms. Livestock and carnivorous have an important role in transporting sarcocystosis in humans. In a previously study, we detected that $100 \%$ of 100 sheep and cattle samples had been infected by Sarcocystis species using digestion and microscopic method (21). For identifying species of Sarcocystis, 50 samples of sheep and cattle samples (each one 25 samples) were selected to determinate species of the parasite using the PCR-RFLP method. The reason of performing this study was to distinguish the risk of human sarcocystosis due to consumption of contaminated meat as a public health problem.

Currently, the identification of Sarcocystis species in animals and humans is carried out by using transmission electron microscopy to study structure of cyst wall (22). However, the use of this method has some limitations in the extended epidemiology study and detection of little morphology variation in species (23). Therefore, many investigators use the molecular approach for identification of Sarcocystis species variation. At this point, 2 genes were presented, which included $18 \mathrm{~S}$ ribosomal RNA and small subunit ribosomal RNA gene. In this study we used $18 \mathrm{~S} r$ RNA gene for distinguishing Sarcocystis species in studied animals. We find that all Sarcocystis isolates in cattle sam- ples belonged to S. cruzi. The results of the PCR of cattle samples presented a 600-nucleotide bp fragment. Comparison of studied nucleotide sequencing with other sequence of Sarcocystis species in the gene bank revealed 99\% homology with $S$. cruzi with only 2 nucleotide different (Figure 2).

The nucleotides sequence of $S$. cruzi was booked to the NCBI gene bank as LC214880, LC214881, LC214882, and LC214883 accession numbers. This finding should be considered by the veterinary organization due to the fact that S. cruzi has severe pathogenicity in livestock and can cause severe clinical sign, abortion, and loss of animal products, however, without any pathologic effects on the human population. Controversy, Agholi et al. described that $S$. cruzi was detected in fecal samples of one women immunocompromised patient (HIV positive) using 18S r DNA gene amplifying and phylogenic analysis (24). The S. cruzi has worldwide distribution and is frequently reported. Sarcocystiscruzi, S. hirsute, and S. hominis cysts were detected on imported cattle meat from Argentina to Norway in 2009 (25). Pritt et al. showed that 31 samples of 48 (64.5\%) beef meat samples are infected by $S$. cruzi using the molecular and histology method and indicated high prevalence $S$. cruzi in USA. More et al. in Germany, indicated that 52\% of 275 beef samples were infected by S. cruzi, following $37 \%$ by S. sinensis (26). Additionally, water buffalo can also serve as an intermediated host for S. cruzi $(27,28)$. In Iran, there are many articles confirmed that the predominant species of Sarcocystis in cattle is S. cruzi (29-31).

The present study indicated that all sheep samples were predominantly infected by S. tenella (80\%) and followed by S. capracanis (20\%). Amplifying of the18S r RNA gene by PCR showed a 1100 and 900 bp nucleotide segments for $S$. tenella and $S$. capracanis, respectively. The comparison of nucleotide sequence with other booked nucleotide in the gene bank revealed that there are > 99\% homology with 2 mentioned Sarcocystis species with 5 different nucleotide for S. capracanis (Figure 7). Sarcocystis tenella is one of the pathogen Sarcocystis species in sheep. The abortion in sheep currently happened in this area and it seems that $S$. tenella should be considered as important agents for abortion and loses of animal production. Three restricted enzymes included EcoR1, Mbo, and Hinf can break down the PCR yield to 2 or 3 fragments. The PCR and RFLP analyzing of sheep samples showed that 2 species of Sarcocystis, S. tenella and S. capracanis, exists in this area.

In this study, we have not seen any macroschizont on sheep carcasses caused by S. gigantea. Controversy, Aghaeipour et al. found no microschizont cyst of S. tenella or S. capracanis in goat, however, they presented macroschizont from $S$. moulei in Tehran and Ghazvin province of Iran (32). Bittencourt et al. found that $95.8 \%$ of sheep and 


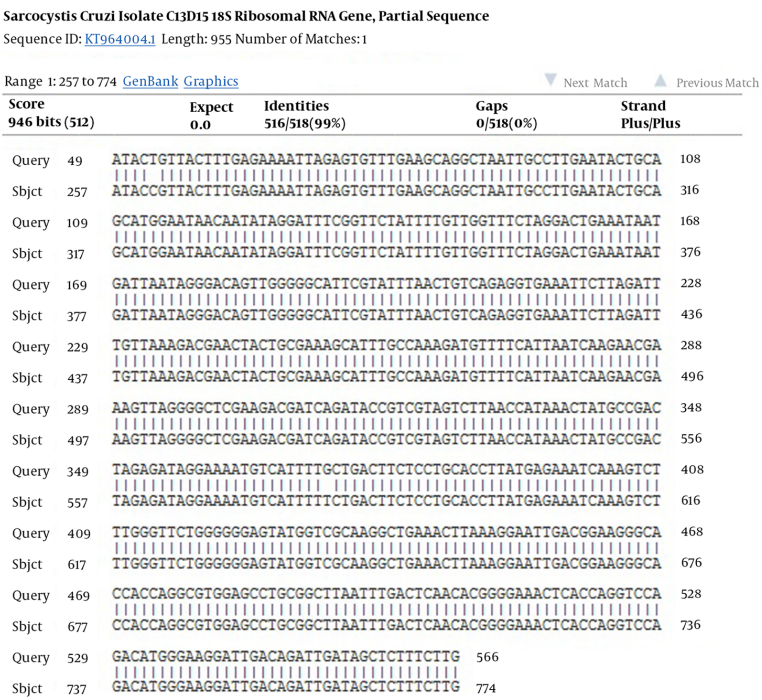

Figure 2. The Comparison of Nucleotide Sequence of Isolated S. cruzi with NCBI Information (99\% Homology)

Figure 3. The Use of Restricted Enzymes on PCR Products from S. cruzi Nucleotide
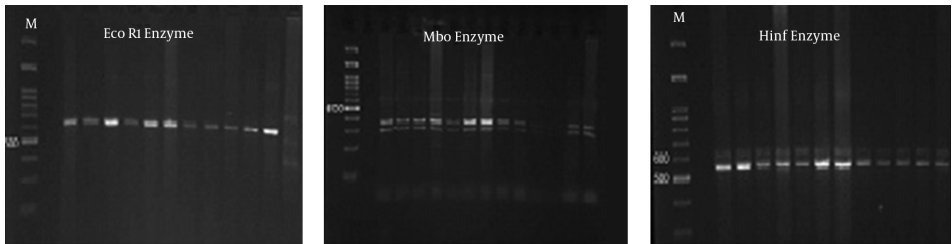

Eco1 (Left) without effect, Mbo enzyme break down to 2 fractions 300 and 350 base pair (Middle) and Hinf enzyme effect with 2 fragment 550 and 50 bp.(Right).

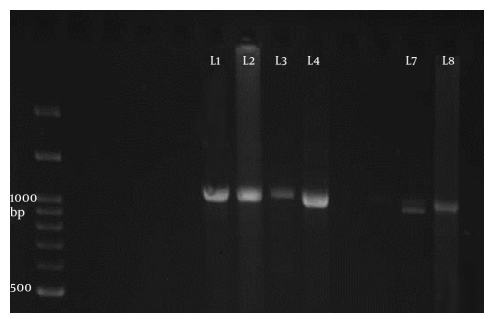

Figure 4. The Electrophoresis of the PCR Product of $18 \mathrm{~S}$ Ribosomal Gene of Sarcocystis in Sheep Samples Presented 2 Bands 1100 (Line 1 - 4) and 900 bp (Line 7 - 8) belonged to S. tenella and S. capracanis, Respectively.

91.6\% of goats in Brazil are infected by S. tenella, S. arieticanis, and S. capracanis, respectively. They reported that the macroschizont, due to S. gigantea or S. medusiformis, was very rare in Brazil (33). Dubey et al. presented that S. arieticanis and S. capracanis are main Sarcocystis species in sheep, in USA. Farhang-Pajuh et al. reported that 29.3\% and $7.52 \%$ of sheep were infected by S. gigantea and S. medusiformis, respectively, using the RFLP-PCR method.

The relationship phylogeny between some species of Sarcocystis in the gene bank was compared with isolated Sarcocystis in the current study by drawing a phylogeny tree (Figure 7). The phylogeny finding presented that there is a $99 \%$ relationship between isolated S. tenella and $92 \%$ between S. capricanis. The relationship between S. cruzi and $S$. tenella was 69\%. Other Sarcocystis species showed various relationships (Figure 7). This study showed that Sarcocystis species in infected sheep and cows in this area are not pertained to human Sarcocystis species and cannot induce sarcocystosis in human population. Further studies on patients with gastrointestinal symptoms such as persistence diarrhea, especially in immunocompromised patients are needed.

\subsection{Conclusions}

In this study we presented that all isolated Sarcocystis species in cattle and sheep belonged to S. cruzi, S. tenella, 
Figure 5. The Use of Restricted Enzymes on PCR Yields of S. tenella Nucleotide in Sheep Samples
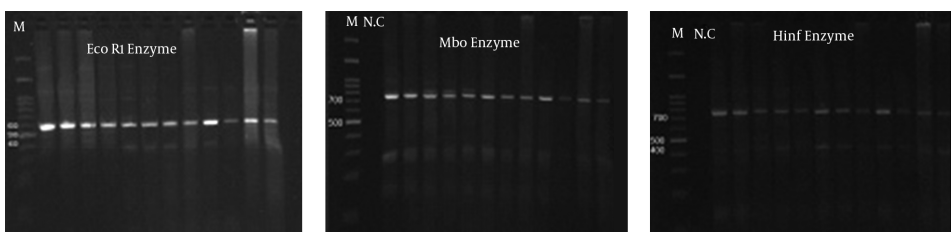

Eco1 enzyme breakdown to 600 and 400 bands (Left), Mbo with 3 fractions 750, 250, and 150 (Middle) and hinf with 2 fragment 750 and 400 (Right).



Figure 6. The Comparison of the Nucleotide Sequence of S. capracanis with NCBI Information (99\% Homology)

Figure 7. The Comparison of Sarcocystis Species Relationship Phylogeny

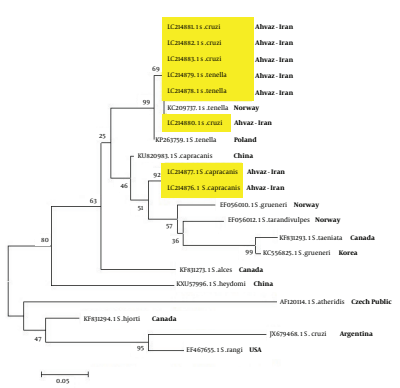

(The isolated current samples have been highlighted). and S. capracanis in this area and none of them have an important role for transmission human sarcocystosis. However, S. cruzi and S. tenella, as pathogen species, should be considered for causing economic loss in livestock animals by veterinary office.

\section{Acknowledgments}

Ahvaz Jundishapur University of Medical Sciences financially supported this study with grant number: 90111.

\section{Footnotes}

Authors' Contribution: Mahmoud Rahdar: planning, sample collection, bioinformatics analysis, writing; 
Tahereh Kardooni: performing molecular technique PCR-RFLP.

\section{Conflict of Interest: There is no any conflict of interest.}

\section{References}

1. Dubey JP, Speer CA, Fayer R. Sarcocystosis of animals and man. CRC Press, Inc.; 1989.

2. Tenter AM, Johnson AM. Phylogeny of the tissue cyst-forming coccidia. Adv Parasitol. 1997;39:69-139. [PubMed: 9241815].

3. Fayer R, Esposito DH, Dubey JP. Human infections with Sarcocystis species. Clin Microbiol Rev. 2015;28(2):295-311. doi: 10.1128/CMR.0011314. [PubMed: 25715644].

4. Tenter AM. Current research on Sarcocystis species of domestic animals. Int J Parasitol. 1995;25(11):1311-30. [PubMed: 25715644].

5. Soulsby EJL. Helminths, arthropods and protozoa of domesticated animals. Bailliere Tindall; 1982.

6. Jeffrey M. Sarcocystosis of sheep. In Practice. 1993;15(1):2-8. doi: 10.1136/inpract.15.1.2.

7. Fayer R, Dubey JP. Sarcocystis induced abortion and fetal death. Prog Clin Biol Res. 1988;281:153-64. [PubMed: 3140250].

8. O'Donoghue P, Rommel M. Australian-German collaborative studies on the immunology of Sarcocystis infections. Angew Parasitol. 1992;33(2):102-19. [PubMed: 1610015].

9. Martínez-Navalon B, Anastasio-Giner B, Cano-Fructuoso M, SanchezMartínez P, Llopis-Morant A, Perez-Castarlenas B, et al. Sarcocystis infection: a major cause of carcass condemnation in adult sheep in Spain. Spanish J Agric Res. 2012;10(2):388-92.

10. Arness MK, Brown JD, Dubey JP, Neafie RC, Granstrom DE. An outbreak of acute eosinophilic myositis attributed to human Sarcocystis parasitism. Am J Trop Med Hyg. 1999;61(4):548-53. [PubMed: 10548287].

11. Mehrotra R, Bisht D, Singh PA, Gupta SC, Gupta RK. Diagnosis of human sarcocystis infection from biopsies of the skeletal muscle. Pathology. 1996;28(3):281-2. [PubMed: 8912363].

12. Tappe D, Abdullah S, Heo CC, Kannan Kutty M, Latif B. Human and animal invasive muscular sarcocystosis in Malaysia-recent cases, review and hypotheses. Trop Biomed. 2013;30(3):355-66. [PubMed: 24189667].

13. Italiano CM, Wong KT, AbuBakar S, Lau YL, Ramli N, Omar SFS, et al. Sarcocystis nesbitti causes acute, relapsing febrile myositis with a high attack rate: description of a large outbreak of muscular sarcocystosis in Pangkor Island, Malaysia, 2012. PLoS Neglect Trop Dis. 2014;8(5):e2876. .

14. Esposito DH, Stich A, Epelboin L, Malvy D, Han PV, Bottieau E, et al. Acute muscular sarcocystosis: an international investigation among ill travelers returning from Tioman Island, Malaysia, 2011-2012. Clin Infect Dis. 2014;59(10):1401-10. doi: 10.1093/cid/ciu622. [PubMed: 25091309].

15. Yang ZQ, Li QQ, Zuo YX, Chen XW, Chen YJ, Nie L, et al. Characterization of Sarcocystis species in domestic animals using a PCR-RFLP analysis of variation in the 18S rRNA gene: a cost-effective and simple technique for routine species identification. Exp Parasitol. 2002;102(34):212-7. [PubMed: 12856319].

16. Yang ZQ, Zuo YX, Chen XW, Ding B, Luo J, Zhang YP. 18S rRNA gene of Sarcocystis hominis cyst from water buffalo and cattle. Zool Res. 2000;21(2):133-8.

17. Fischer S, Odening K. Characterization of bovine Sarcocystis species by analysis of their $18 \mathrm{~S}$ ribosomal DNA sequences. J Parasitol. 1998;84(1):50-4. [PubMed: 9488337].
18. Pritt B, Trainer T, Simmons-Arnold L, Evans M, Dunams D, Rosenthal BM. Detection of sarcocystis parasites in retail beef: a regional survey combining histological and genetic detection methods. J Food Prot 2008;71(10):2144-7. [PubMed: 18939769].

19. Rahdar M, Salehi M. The prevalence of sarcocystis infection in slaughtered cattle and sheep using digestion method in ahvaz city of iran. Biochem Cell Arch. 2011;11(2):469-71.

20. Wong KT, Pathmanathan R. Ultrastructure of the human skeletal muscle sarcocyst. J Parasitol. 1994;80(2):327-30. [PubMed: 8158479].

21. McManus DP, Bowles J. Molecular genetic approaches to parasite identification: their value in diagnostic parasitology and systematics. Int J Parasitol. 1996;26(7):687-704. [PubMed: 8894760].

22. Agholi M, Shahabadi SN, Motazedian MH, Hatam GR. Prevalence of Enteric Protozoan Oocysts with Special Reference to Sarcocystis cruzi among Fecal Samples of Diarrheic Immunodeficient Patients in Iran Korean J Parasitol. 2016;54(3):339-44. doi: 10.3347/kjp.2016.54.3.339. [PubMed: 27417091].

23. Gjerde B. Phylogenetic relationships among Sarcocystis species in cervids, cattle and sheep inferred from the mitochondrial cytochrome c oxidase subunit I gene. Int J Parasitol. 2013;43(7):579-91. doi:10.1016/j.ijpara.2013.02.004. [PubMed: 23542092].

24. More G, Pantchev A, Skuballa J, Langenmayer MC, Maksimov P, Conraths FJ, et al. Sarcocystis sinensis is the most prevalent thick-walled Sarcocystis species in beef on sale for consumers in Germany. Parasitol Res. 2014;113(6):2223-30. doi: 10.1007/s00436-014-3877-x. [PubMed 24700022]

25. Li QQ, Yang ZQ, Zuo YX, Attwood SW, Chen XW, Zhang YP. A PCRbased RFLP analysis of Sarcocystis cruzi (Protozoa: Sarcocystidae) in Yunnan Province, PR China, reveals the water buffalo (Bubalus bubalis) as a natural intermediate host. J Parasitol. 2002;88(6):125961. doi: 10.1645/0022-3395(2002)088[1259:APBRAO]2.0.CO;2. [PubMed: 12537125].

26. Jehle C, Dinkel A, Sander A, Morent M, Romig T, Luc PV, et al. Diagnosis of Sarcocystis spp. in cattle (Bos taurus) and water buffalo (Bubalus bubalis) in Northern Vietnam. Vet Parasitol. 2009;166(3-4):314-20. doi: 10.1016/j.vetpar.2009.08.024. [PubMed: 19783101].

27. Hajimohammadi B, Eslami G, Zohourtabar A, Dehghani A, Oryan A, Tafti H. High Occurrence of Sarcocystis Cysts in Meat Produced in Yazd, Central Iran.J Food Qual Hazards Control. 2014;1(4):95.

28. Nourollahi Fard SR, Asghari M, Nouri F. Survey of Sarcocystis infection in slaughtered cattle in Kerman, Iran. Trop Anim Health Prod. 2009;41(8):1633-6. doi: 10.1007/s11250-009-9358-z. [PubMed: 19390981].

29. Nourollahi-Fard SR, Kheirandish R, Sattari S. Prevalence and histopathological finding of thin-walled and thick-walled Sarcocysts in slaughtered cattle of Karaj abattoir, Iran. J Parasit Dis 2015;39(2):272-5. doi: 10.1007/s12639-013-0341-2. [PubMed: 26064016].

30. Motamedi G, Dalimi A, Aghaeipour K, Nouri A. Ultrastructural and molecular studies on fat and thin macrocysts of Sarcocystis spp. isolated from naturally infected goats. Arch Razi Inst. 2010;65(2):91-7.

31. Bittencourt MV, Meneses ID, Ribeiro-Andrade M, de Jesus RF, de Araujo FR, Gondim LF. Sarcocystis spp. in sheep and goats: frequency of in fection and species identification by morphological, ultrastructural, and molecular tests in Bahia, Brazil. Parasitol Res. 2016;115(4):1683-9. doi:10.1007/s00436-016-4909-5. [PubMed: 26786832]

32. Dubey JP, Lindsay DS, Speer CA, Fayer R, Livingston CJ. Sarcocystis arieticanis and other Sarcocystis species in sheep in the United States. Parasitol. 1988;74(6):1033-8. [PubMed: 3142990].

33. Farhang-Pajuh F, Yakhchali M, Mardani K. Molecular determination of abundance of infection with Sarcocystis species in slaughtered sheep of Urmia, Iran. Vet Res Forum. 2014;5(3):181-6. 